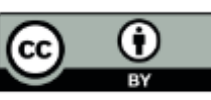

\title{
Experiencias de la misión médica en Colombia. Voces en medio del conflicto armado*
}

\author{
Experiences of the Medical Mission in Colombia. \\ Voices in the middle of the armed conflict
}

Experiências da Missão Médica em

Colômbia. Vozes no meio do conflito armado

Recibido: 09 de Agosto de 2019. Aceptado: 22 de

Enero de 2020. Publicado: 1 de Octubre de 2020.

DOI: https://doi.org/10.11144/Javeriana.rgps19.emmc

\author{
Isabel Cristina Garcés Palacio \\ Universidad de Antioquia, Colombia \\ ORCID: https://orcid.org/0000-0003-0531-276X \\ Sara Milena Ramos Jaraba \\ Universidad de Antioquia, Colombia \\ ORCID: https://orcid.org/0000-0002-8638-5209 \\ Natalia Quiceno Toro \\ Universidad de Antioquia, Colombia \\ ORCID: https://orcid.org/0000-0001-9689-4835 \\ Marlly Andrea García Jiménez \\ Universidad de Antioquia, Colombia \\ ORCID: https://orcid.org/0000-0001-9223-5824 \\ María Ochoa Sierra \\ Universidad de Antioquia, Colombia \\ ORCID: https://orcid.org/0000-0001-7284-5182 \\ Edison Bedoya Bedoya \\ Universidad de Antioquia, Colombia \\ ORCID: https://orcid.org/0000-0001-6499-9800 \\ Laura Estefany Ruiz Sánchez \\ Universidad de Antioquia, Colombia \\ ORCID: https://orcid.org/0000-0002-2126-2451
}

\begin{abstract}
Para citar este artículo Garcés IC, Ramos SM, Quiceno N, García MA, Ochoa M, Bedoya E, Ruiz LE. Experiencias de la misión médica en Colombia. Voces en medio del conflicto armado. Rev Gerenc Polit Salud. 2020;19. https://doi.org/10.11144/Javeriana.rgps19.emmc
\end{abstract}

\footnotetext{
${ }^{\text {a }}$ Autora de correspondencia. E-mail: icristina.garces@udea.edu.co
} 


\section{Resumen}

Objetivo: describir la experiencia de la misión médica en el marco del conflicto armado en dos municipios colombianos. Métodos: investigación cualitativa con enfoque de estudio de caso comparativo a través de 22 entrevistas y 3 grupos focales. Para el tratamiento de la información se utilizaron técnicas del análisis de contenido. Resultados: en el marco del conflicto, en especial por las restricciones de movilidad, se presentaron dificultades para la prestación y el acceso a los servicios de salud, y el acceso a medicamentos. Se reportaron transgresiones a la Misión Médica como infracciones contra la vida y la infraestructura, y violaciones al secreto profesional. Se recomienda durante el post-acuerdo mejorar las condiciones laborales y la formación del personal de salud, incluyendo la capacitación en derecho internacional humanitario. Conclusión: el conflicto armado afectó en forma sustancial al personal de salud y la prestación del servicio.

Palabras clave: Conflictos armados, personal de salud, barreras de acceso a los servicios de salud, análisis cualitativo, Colombia.

\section{Abstract}

Objective: To describe the experience of the medical mission in Colombia in the context of the armed conflict of two municipalities. Methods: qualitative research was done using a comparative case study approach based on 22 interviews and three focus groups. The processing of information was conducted using content analysis techniques. Results: in the context of the conflict, especially due to mobility restrictions, there were difficulties in the provision of and access to health services and medicines. Transgressions to the medical mission, such as infractions against life and infrastructure, as well as violations of professional confidentiality, were reported. In the post-agreement phase, it is recommended to improve the working conditions and health personnel training, especially in International Humanitarian Law. Conclusion: the armed conflict substantially affected the medical staff's health and the provision of health services.

Keywords: Armed conflicts, health personnel, barriers to access of health services, qualitative analysis, Colombia.

\section{Resumo}

Objetivo: descrever a experiência da missão médica no marco do conflito armado em dois municípios colombianos. Métodos: pesquisa qualitativa com enfoque de estudo de caso comparativo a través de 22 entrevistas e 3 grupos focais. Para o tratamento da informação se utilizaram técnicas de análise de conteúdo. Resultados: no marco do conflito, em especial devido às restrições de mobilidade, se presentaram dificuldades para a prestação e aceso aos serviços de saúde, e o aceso aos medicamentos. Reportaram-se transgressões à Missão Médica, tais como infrações contra a vida e a infraestrutura, e violações ao segredo profissional. Recomenda-se durante o pós-acordo melhorar as condições laborais e a formação do pessoal de saúde, incluindo a capacitação em direito internacional humanitário. Conclusão: o conflito armado afeitou em forma substancial ao pessoal de saúde e a prestação do serviço.

Palavras-chave: Conflitos armados, pessoal de saúde, barreiras de aceso aos serviços de saúde, análise qualitativo, Colômbia. 


\section{Introducción}

El conflicto armado colombiano lleva más de cinco décadas y ha dejado 218.094 muertes según el informe Basta Ya (2013) (1). Los Convenios de Ginebra de 1949 y sus Protocolos adicionales, base del Derecho Internacional Humanitario (DIH), son un conjunto de normas jurídicas que pretenden limitar los efectos de los conflictos armados. Dichos convenios están dirigidos a proteger especialmente a las personas que no participan o que ya no pueden seguir participando de las hostilidades o combates, entre estos el personal sanitario y los civiles (2). En Colombia, pese a la firma y ratificación de estos convenios en 1996, solo hasta 2002 el Ministerio de Salud dictó medidas para la protección de la Misión Médica (3). Igualmente, solo hasta 2012 se adoptó el Manual de Misión Médica, a través del cual se regula la señalización y divulgación de su Emblema. Este mecanismo tiene como objetivo facilitar el trasporte de medicamentos e insumos sanitarios, así como la atención de heridos y enfermos en el marco de la misión humanitaria (4).

El Comité Internacional de la Cruz Roja reportó que entre los años 1995 y 1998 las infracciones más recurrentes a la Misión Médica fueron los ataques contra la vida y la integridad del personal, así como los ataques a la infraestructura (5). Posteriormente, entre 2001 y 2004 se presentaron 48 infracciones por restricción y prohibición de oferta sanitaria, 43 casos de robo de medicamentos, 30 casos de ataques a unidades sanitarias, 17 infracciones contra personas y 29 ataques a transportes sanitarios (6). Durante el 2012 se presentaron 75 infracciones contra la Misión Médica, y 13 incidentes que incluyeron amenazas y ataques contra el personal y la infraestructura sanitaria (7).

En Colombia los ataques contra la Misión Médica han sido frecuentes, por lo cual organizaciones como el Comité Internacional de Cruz Roja, el Centro Nacional de Memoria Histórica, el Centro de Investigación y Educación Popular, entre otras, han realizado amplios reportes y seguimientos sobre el tema a lo largo de los últimos 20 años. Estos reportes han evidenciado que el personal de salud ha sido uno de los actores más afectados. Sin embargo, más allá del reporte cuantitativo de los incidentes o infracciones, es importante hacer una lectura más cualitativa sobre lo que implica el acceso a la salud en contextos rurales, rescatando la experiencia y las voces de diversos actores. Especialmente, debe resaltarse lo que implica el trabajo del personal médico en el conflicto armado. Esta necesidad se incrementa en la era post-acuerdo, entendida como una fase posterior en la evolución del conflicto que conduciría idealmente a una situación de posconflicto. En esta fase se esperaría un cambio relacionado con la protección y garantías de seguridad a la Misión Médica. Por lo anterior, a través de este artículo se describe la experiencia del personal de salud, actores comunitarios, funcionarios del gobierno y de Naciones Unidas, en el marco del conflicto armado en Colombia, y en el postacuerdo, en dos municipios colombianos expuestos a diferentes niveles de conflicto.

\section{Metodología}

Se realizó una investigación cualitativa con enfoque interpretativo, a través de un estudio de caso comparativo, utilizando entrevistas y grupos focales (8). El estudio se llevó a cabo entre junio y agosto de 2018, en dos municipios del departamento de Antioquia; uno con altos índices 
de conflicto armado, y el otro con bajos índices. Además, se realizaron algunas entrevistas en Medellín, centro administrativo del Departamento, donde se encuentran funcionarios y organizaciones que han trabajado en estos municipios. El municipio con alta intensidad del conflicto está ubicado en la región occidente, en la cual confluían diversos actores armados legales e ilegales en disputa por el territorio: Guerrillas, Paramilitares, Bandas Criminales, y Fuerzas Militares. El municipio con baja intensidad del conflicto se encuentra ubicado en el suroeste del departamento, y la presencia de los actores armados fue intermitente.

La selección de estos municipios se determinó por la diferencia sustancial en el número de víctimas, las similitudes demográficas (en los dos hay presencia importante de comunidades indígenas), los dos municipios cuentan con hospital de primer nivel, condiciones similares de accesibilidad desde la capital del departamento y las condiciones de seguridad para el equipo de campo. Este artículo se deriva de un proyecto titulado: "Salud en escenarios de conflicto y post-conflicto: salud reproductiva y materna en una Colombia en el camino a la recuperación”, el cual es parte de un proyecto multicéntrico realizado por el Consorcio BRANCH (Bridging Research \& Action in Conflict Settings for the Health of Women \& Children). El estudio se llevó a cabo simultáneamente en 10 países en Conflicto: Siria, Yemen, República del Congo, Afganistán, Pakistán, Sudán del Sur, Nigeria, Somalia y Colombia.

\section{Recolección de información y participantes}

Se realizaron 22 entrevistas y tres grupos focales. Los participantes incluyeron: personal de salud, personal de instituciones gubernamentales, de varias $\mathrm{ONG}$, de la Organización de Naciones Unidas (ONU), además de representantes de organizaciones comunitarias e indígenas en los dos municipios de estudio, Medellín y Bogotá. Los participantes se seleccionaron teniendo en cuenta que hubieran experimentado o tuvieran conocimiento acerca de la prestación de servicios de salud durante el conflicto armado en Colombia y/o durante el post-acuerdo.

Las entrevistas y grupos focales fueron grabados en audio y posteriormente transcritos. Para ambas técnicas de recolección se utilizaron guías semiestructuradas de acuerdo con el perfil del entrevistado (una para personal de salud, una para funcionarios del gobierno, otra para funcionarios de la ONU y otra para actores comunitarios). Dichas guías fueron proporcionadas por el Consorcio BRANCH y adaptadas para el contexto colombiano. Mediante las guías se busca analizar tres aspectos centrales durante el conflicto y el post-acuerdo: los programas o proyectos, la prestación y acceso a servicios de salud y personal de salud, y el contexto de intervención. Para este artículo se establecieron dos categorías analíticas: servicios de salud y personal de salud en contextos de conflicto y post-acuerdo. 


\section{Análisis de los datos}

Para el análisis de los datos, cinco investigadoras realizaron la codificación manual de forma independiente, buscando reducir los datos por medio de la segmentación de textos transcritos. Para esto se diseñó una matriz de Excel, con las categorías y subcategorías en las columnas, y los entrevistados, los códigos y abreviaciones en las filas. Esta forma de codificación permitió el análisis y la interpretación de diversas relaciones generadas al interior de la matriz. De esta manera, se observaron diferencias y semejanzas entre los relatos de los actores y entre los municipios. Posterior a este proceso, se utilizaron técnicas de análisis de contenido (9), para reducir la información en un solo documento agrupando códigos en relación a tres subcategorías emergentes: barreras en el acceso y prestación de servicios de salud en el conflicto, transgresiones a la Misión Médica, y recomendaciones del post-acuerdo en materia de atención y personal en salud.

\section{Consideraciones éticas}

Este estudio fue aprobado por el comité de ética de la Facultad Nacional de Salud Pública de la Universidad de Antioquia, el comité de ética de la Universidad de Harvard T. H. Chan School of Public Health, y el Hospital for Sick Children. Los participantes firmaron un consentimiento informado. Se respetó su participación voluntaria y su confidencialidad utilizando códigos. Además, por recomendación del comité no se presentan los nombres de los municipios con el fin de proteger la identidad de los participantes. Por otro lado, y como parte de la ética del proceso investigativo, se hicieron ejercicios de socialización de lo encontrado con actores claves en los municipios, además de un evento de carácter general y ampliado en la Universidad de Antioquia, con participación de personal del Ministerio de la Salud y de la Secretaría de Salud de Antioquia.

\section{Resultados}

Participaron 27 personas, 12 hombres y 15 mujeres. En el perfil correspondiente a personal de salud participaron nueve profesionales de la salud: enfermería, medicina, y psicología, un auxiliar de enfermería, y un vacunador. Entre los actores gubernamentales, fueron entrevistados: un miembro de la Secretaría de Salud Departamental y tres de la Secretaría de Salud Municipal. Del sector académico participaron cuatro profesores de universidades públicas y privadas, tres representantes de ONG, y cuatro representantes de Agencias de Naciones Unidas en Colombia. Participaron además, por parte de las organizaciones: dos miembros de una organización de víctimas y uno de una comunidad indígena.

La información recolectada da cuenta de una amplia diversidad temporal, pues se indagó a los participantes por hechos que ocurrieron en los años de mayor intensidad del conflicto (1996-2005), y durante los diálogos de paz y el post-acuerdo (2012-2018). La suma de estas dos periodizaciones es de aproximadamente veinte años. Los resultados dan cuenta de las dificultades que atraviesa el personal de salud en el municipio de alta conflictividad, en contraposición con el de baja conflictividad. Debido a que la presencia de actores armados en 
el municipio de baja conflictividad fue leve e intermitente, la Misión Médica no sufrió grandes afectaciones, ni tuvo barreras sustanciales para ejercer sus funciones. En cambio, en el municipio de alta conflictividad, las afectaciones a la comunidad, al personal sanitario y a la infraestructura fueron substantivas, y se dieron con mayor ímpetu en el periodo comprendido entre $1995 \mathrm{y}$ 2005. Dichas afectaciones no han cesado a la fecha, por lo cual se dará mayor énfasis a los testimonios relativos a este municipio del occidente Antioqueño.

\section{Barreras en el acceso y prestación de servicios de salud en el conflicto}

Los participantes de la investigación manifestaron que el conflicto armado en Colombia, si bien se ha presentado en casi la totalidad del territorio nacional, fue más intenso en las áreas rurales/ dispersas, en las cuales adicionalmente existen barreras estructurales como el acceso geográfico; así como "barreras institucionales, socioeconómicas, y culturales que están asociadas a todo el tema de salud pública, y que se hace más evidente en estos territorios" (funcionario de la ONU). Aunadas al conflicto, tales barreras crean mayores dificultades para acceder a los servicios de salud. Esto es, en todo caso, mayor en el municipio de alta conflictividad, puesto que las zonas de mayor dificultad de acceso son aquellas en las que hay mayor presencia de actores armados. En estos municipios es común que la población termine confinada y aislada, imposibilitando a la Misión Médica prestar los servicios de salud.

Frente a la complejidad geográfica y territorial, en el país se ha utilizado la estrategia de equipos de salud extramurales para acercar los servicios a las comunidades más alejadas del casco urbano. Estos servicios de atención básica se dedican por lo general a realizar actividades de promoción de la salud, prevención de la enfermedad y a llevar medicamentos y métodos anticonceptivos. Los participantes mencionaron que, debido a las dinámicas del conflicto, los hospitales suspendieron los servicios de salud extramurales. Solo en casos graves de epidemias y mortalidad por alguna enfermedad se solicitaba autorización a los grupos armados para acceder a las zonas. La ausencia de estas jornadas generó una percepción de abandono por parte del Estado e incredulidad frente a los servicios de salud. Por el contrario, en el municipio de baja conflictividad, las jornadas no solo no cesaron, sino que se prorrogaron, de modo que la población tuvo atención constante.

Los participantes del municipio de alta conflictividad refirieron que las distintas formas de restricción de la movilidad fueron una de las mayores barreras para acceder a los servicios de salud. Debido a retenes de grupos armados, o a la presencia de minas antipersona, las comunidades quedaban atrapadas o con movilidad restringida a determinados momentos u horas del día. Esto implicó muchas veces el confinamiento de la población: "ni salían ni entraba porque a uno le daba miedo salir al pueblo y los del pueblo les daba miedo salir al campo" (Miembro de organización de víctimas). Lo anterior creaba una percepción de inseguridad, temor y zozobra. Por este motivo, algunas comunidades rurales quedaron aisladas, sin los servicios básicos, entre ellos salud, alimentación adecuada, etc. Algunos testimonios reportan que, en el municipio de 
mayor conflictividad, las comunidades de las veredas más lejanas pasaron alrededor de cinco años sin recibir servicios de salud, siendo atendidos solo en ocasiones por la ONG Médicos Sin Fronteras.

Los retenes armados como una forma de restricción a la movilidad obstaculizaron la prestación de los servicios de salud en zonas rurales: "no podía ni entrar ni salir nadie" (Miembro de organización de víctimas). En municipios con presencia de diversos grupos, la situación era alarmante pues existía disputa por el control de los territorios y se estigmatizaba a los pobladores como colaboradores de su adversario al pasar de una zona controlada por un grupo a otra. Esta situación también se repitió de manera regular con los trabajadores de la salud.

Como consecuencia del confinamiento, los retenes, la estigmatización de la población y la suspensión de los servicios extramurales, las comunidades crearon estrategias informales para suplir la falta de atención. Entre estas se encuentran el uso de la medicina tradicional, así como los saberes de médicos ancestrales, familiares y vecinos. Las atenciones más comunes se relacionan con enfermedades gastrointestinales, respiratorias, atención del parto y a los heridos a raíz del conflicto.

\section{Transgresiones a la Misión Médica}

Los entrevistados develaron que el conflicto armado colombiano tuvo consecuencias sobre la Misión Médica, poniendo en riesgo la vida e integridad del personal, además de las afectaciones a la infraestructura sanitaria. Estos delitos tipificados por el DIH y contemplados en los manuales de Misión Médica se expondrán a continuación.

\section{Infracciones contra la vida y la integridad}

Durante el conflicto armado, el personal de salud experimentó situaciones de temor, angustia, lesiones personales, amenazas, secuestros e incluso la muerte. Esto sucedió en mayor proporción en aquellas zonas donde varios grupos armados se disputaban un territorio. Estas afectaciones, muchas veces fueron indirectas, cuando los grupos armados tomaban medidas de represión, amenaza o control en las poblaciones aledañas. Otras veces fueron directas, cuando el propio personal fue víctima de violencia verbal o física. Ver la tabla 1. 
Isabel Cristina Garcés Palacio/ Sara Milena Ramos Jaraba/ Natalia Quiceno Toro/ Marlly Andrea García Jiménez/ et al.

Tabla 1 Infracciones contra la vida de la misión médica en el conflicto armado (Cont.)

\begin{tabular}{|c|c|}
\hline Tipología & Relato \\
\hline Homicidio & $\begin{array}{l}\text { Alta conflictividad: } \\
\text { "Soy testigo del asesinato del gerente del hospital en el que trabajaba, del } \\
\text { médico, director del centro de salud" (Profesional Académico). } \\
\text { "Al primito mío (promotor de salud) lo mataron, a todos los mataron porque } \\
\text { decían que colaboraban con la guerrilla" (Víctima del Conflicto). } \\
\text { Baja conflictividad: No aplica. }\end{array}$ \\
\hline Desaparición forzosa & $\begin{array}{l}\text { Alta conflictividad: } \\
\text { "El (promotor) viniendo del pueblo lo dejaron en el retén, él quedó } \\
\text { desaparecido, nunca lo encontraron" (Victima del Conflicto). } \\
\text { Baja conflictividad: No aplica. }\end{array}$ \\
\hline Lesiones personales & $\begin{array}{l}\text { Alta conflictividad: } \\
\text { "En esa semana cogieron un médico y una auxiliar de enfermería, y había } \\
\text { habido un enfrentamiento, tenían muchos heridos, eso fue como del } \\
\text { bombardeo que hubo, hubo muchos heridos y ahí resultó el médico y la } \\
\text { auxiliar de recursos humanos" (Vacunador). } \\
\text { Baja conflictividad: No aplica. }\end{array}$ \\
\hline Amenazas & $\begin{array}{l}\text { Alta conflictividad: } \\
\text { "Las directivas que estaban aquí, más desprotegidos que un verriondo, } \\
\text { porque él se fue atender desde Medellín y el alcalde también, eso porque los } \\
\text { paracos volvieron a entrar y lo hicieron venir" (Vacunador). } \\
\text { Baja conflictividad: No aplica. }\end{array}$ \\
\hline Miedo & $\begin{array}{l}\text { Alta conflictividad: } \\
\text { "Vimos un helicóptero, al compañero que estaba allá lo bombardearon, él se } \\
\text { tuvo que tirar para una calle y esconder, yo en ese momento venía con mi } \\
\text { otro compañero y le decía, hermano aquí nos tocó" (Vacunador). } \\
\text { Baja conflictividad: No aplica. }\end{array}$ \\
\hline $\begin{array}{l}\text { Desplazamiento } \\
\text { forzado }\end{array}$ & $\begin{array}{l}\text { Alta conflictividad: } \\
\text { "Los hicieron desplazar y sí, hubo a unos que, si los amenazaron, hasta la } \\
\text { amenaza, pero de muerte" (Vacunador). } \\
\text { Baja conflictividad: No aplica. }\end{array}$ \\
\hline $\begin{array}{l}\text { Secuestro y toma de } \\
\text { rehenes }\end{array}$ & $\begin{array}{l}\text { Alta conflictividad: } \\
\text { "Algo que nos pasó a todos en el hospital, donde llegó alguien haciéndose } \\
\text { pasar por la guerrilla, era o no era guerrillero y nos encerró aquí a todos... } \\
\text { un montón de horas, con pistola, se robó algunas cadenas, yo me logré salir } \\
\text { por aquí fui por la policia, pero la policía tenía más miedo que todo mundo, } \\
\text { la policía no salía porque la guerrilla estaba por todos los lados y ellos no } \\
\text { pasaban del parque entonces se los llevó por allá y después lo soltó" } \\
\text { (Médico). } \\
\text { Baja conflictividad: No aplica. }\end{array}$ \\
\hline
\end{tabular}

Fuente: elaboración propia. 
Tabla 1 Infracciones contra la vida de la misión médica en el conflicto armado (Cont.)

\begin{tabular}{|l|l|}
\hline $\begin{array}{l}\text { Falta de } \\
\text { disponibilidad de } \\
\text { personal médico en } \\
\text { conflicto }\end{array}$ & $\begin{array}{l}\text { Alta conflictividad: } \\
\text { "Cuando mataron al médico del centro de salud, nosotros nos quedamos sin } \\
\text { médico cuatro meses porque evidentemente nadie va a querer... había una } \\
\text { dificultad en algunas partes del país para conseguir personal sanitario que } \\
\text { vaya y trabaje en zonas donde el conflicto representa una amenaza real para } \\
\text { la vida" (Gerente de hospital/Enfermero). } \\
\text { Baja conflictividad: No aplica. }\end{array}$ \\
\hline $\begin{array}{l}\text { Falta de medidas de } \\
\text { seguridad del personal } \\
\text { de salud }\end{array}$ & $\begin{array}{l}\text { Alta conflictividad: } \\
\text { "Nosotros... hemos viajado en transporte público, en bus, con un carnet y } \\
\text { un chaleco que, ida un calor! en esos municipios del bajo cauca, ¿quién se } \\
\text { pone ese chaleco? cosas muy mal pensabas, muy mal diseñadas y que por } \\
\text { más que nosotros haciamos bulla, no hay quien le importe la seguridad de } \\
\text { uno como profesional" (Psicóloga). } \\
\text { Baja conflictividad: } \\
\text { "No, yo ya después andaba con el uniforme pues de Cruz Roja, y pues } \\
\text { obviamente reporté eso a Cruz Roja, y bajaba acompañado y ya me tocó } \\
\text { cambiar el horario de allá, o sea, máximo me dejaban quedar hasta las 6 de } \\
\text { la tarde, y ya me venia pues" (Enfermero). }\end{array}$ \\
\hline $\begin{array}{l}\text { Estrategias de } \\
\text { atención utilizadas }\end{array}$ & $\begin{array}{l}\text { Alta conflictividad: } \\
\text { "Las medidas que nosotros tomamos de seguridad, es que son los contactos } \\
\text { habituales con los lideres de la comunidad los que te dicen si no hay } \\
\text { peligro, si se puede pasar, con las instituciones y yo creo que la mayor } \\
\text { seguridad es un contacto continuo con todos estos diferentes actores. ONG } \\
\text { que son los que te dan el poder percibir y ponemos a los equipos en terreno } \\
\text { con una cierta garantia. Inmunidad no existe, desafortunadamente en estos } \\
\text { momentos no se puede hablar de inmunidad para ninguna organización" } \\
\text { (Funcionaria de ONG). } \\
\text { Baja conflictividad: No aplica. }\end{array}$ \\
\hline
\end{tabular}

Fuente: elaboración propia.

En el municipio de baja conflictividad solo se reportó una situación en la cual un grupo armado retuvo al vacunador para hacerle preguntas y conocer sus rutas por un tiempo no mayor a diez minutos. Posteriormente, el profesional pudo seguir realizando sus funciones con normalidad. Esta situación propició un ambiente de zozobra, sospecha y vigilancia. "Dentro del conflicto todos los actores están nerviosos de quienes entran a sus zonas de dominio, entonces mientras investigaban quien era y toda la cosa pues los retenían un rato" (Funcionaria de Gobierno). Otras retenciones tenían como propósito apoderarse de las medicinas e insumos, o incluso forzar la atención médica para el grupo armado. Ante esto, el personal comentó ser imparcial y darles trato como sujetos de derecho, lo que no excluía situaciones posteriores de estigmatización por parte de otro actor armado. 
Era común que el personal de salud sólo pudiera acceder a ciertos territorios con permiso de los grupos armados o por solicitud de ellos. Los grupos armados que ejercían el control territorial les daban instrucciones a los profesionales de salud para el ingreso. Estas debían ser cumplidas como ellos lo establecían. "No, hoy no pueden porque vamos a hacer una actividad nosotros. No pueden estar después de tal hora en la zona, no puede salir antes de..." (Enfermera Jefe). Algunos abandonaron su trabajo por presión y amenazas contra su vida. Ya en menor medida, fueron relatados casos de homicidios y lesiones. "... si hubo a unos que sí los amenazaron, hasta la amenaza pero muerte, muerte, pocos" (Vacunador). Las situaciones de inseguridad afectaron también a los promotores de salud rural (actores locales encargados de algunos servicios de salud básica en sus territorios), quiénes fueron asesinados o desaparecidos al etiquetarlos como colaboradores de diversos grupos que se disputaban el control territorial. En el municipio de baja conflictividad no se reportaron este tipo de casos.

Las situaciones anteriormente descritas crearon entre los profesionales un miedo generalizado que interfirió en los servicios prestados en las instituciones de salud. Se presentó una alta rotación del personal, puesto que muchos se negaron a prestar servicios en lugares donde el conflicto armado estaba en apogeo. En cambio, en el municipio de baja conflictividad, el personal de salud es estable. Muchos de ellos llevan más de diez años ejerciendo su labor, además de ser una plaza de trabajo deseada.

Por otro lado, pese a que la presencia de los funcionarios de Naciones Unidas y ONG es mayor en el municipio de alta conflictividad y no en el otro (donde es casi nula), no fueron reportados hechos como los manifestados por el personal de salud. Los participantes de la ONU relatan que esto puede deberse al blindaje internacional que tienen estas organizaciones en los territorios, a los fuertes estudios de seguridad que hace la oficina central, así como al entrenamiento que reciben en Misión Médica. En contraste, las capacitaciones al personal colombiano son escasas, "tristemente [Colombia] no tiene mucho trabajo en el tema de Misión Médica y es por eso que se ha violado mucho" (Profesional Académico). El personal de salud entrevistado en el municipio de alta conflictividad manifestó estar poco entrenado en este tema, por lo cual se sienten inseguros, temerosos y expuestos. Ellos consideran que están siendo descuidados por las entidades de salud y que las medidas de seguridad son mínimas. A pesar de esto, los participantes reportaron que utilizaron algunas medidas de seguridad para salvaguardar su vida o la de su equipo. En ocasiones, estas medidas se realizaban mediante protocolos y entrenamientos discontinuos, proporcionados únicamente por organizaciones no gubernamentales como la Cruz Roja Internacional, Médicos sin Fronteras, o replicados por personal con experiencia previa de trabajo en contextos de conflicto. En otras ocasiones, los participantes hicieron alusión al acompañamiento de diversas ONG a zonas remotas con presencia de grupos armados, lo que servía como garantía para una mejor accesibilidad. 
Isabel Cristina Garcés Palacio/ Sara Milena Ramos Jaraba/ Natalia Quiceno Toro/ Marlly Andrea García Jiménez/ et al.

Otros participantes mencionaron la neutralidad en la prestación del servicio como, por ejemplo, no hablar de temas políticos, ni del conflicto armado con nadie, y muchas veces evitar el contacto con otras personas dentro y fuera de su horario laboral.

Otra estrategia, utilizada en mayor medida por las agencias de Naciones Unidas y ONG, consistía en identificar los grupos armados involucrados, la presencia de la institucionalidad como parte del sistema de seguridad y la constante comunicación con los líderes y organizaciones locales. Los entrevistados afirmaron que mantener una relación de confianza con la comunidad, además del contacto con integrantes de los grupos armados para informar de su visita facilitaba el trabajo extramural.

El personal del municipio de baja conflictividad, en cambio, se siente seguro en su territorio, refiriendo complicaciones de otro orden como la drogadicción, el VIH y el embarazo adolescente como sus principales problemáticas en materia de salud pública. El municipio atiende dichas problemáticas con programas que dependen de los lineamientos del Plan de Desarrollo del alcalde de turno.

\section{Infracciones contra la infraestructura y las actividades sanitarias}

Las infracciones contra la infraestructura fueron menos descritas por los participantes. Sin embargo, en el municipio de alta conflictividad fueron mencionados especialmente el ataque contra medios de transporte sanitarios, y el uso de unidades sanitarias por parte de grupos armados para resguardarse. Estas situaciones impedían que las poblaciones alejadas no pudieran acceder al servicio de ambulancia en caso de urgencias y que los pobladores utilizaran sus propios medios para llegar a los servicios de salud. En muchos casos, esto implicó que se agravaran situaciones reparables: "las sacaban en camilla y no alcanzaban a llegar al pueblo y se morían en el camino" (Miembro de organización de víctimas).

También fueron frecuentes otro tipo de afectaciones tales como forzar la atención médica en condiciones inadecuadas, robos, saqueos y la restricción al tránsito de medicamentos e insumos. Los participantes relatan que estas situaciones se presentaban especialmente en las visitas extramurales, en las que eran interceptados por los grupos armados, quienes les obligaban a atender emergencias médicas y a entregar los medicamentos. Ver la tabla 2. 
Experiencias de la misión médica en Colombia. Voces en medio del conflicto armado

Tabla 2 Infracciones contra la infraestructura y las actividades sanitarias en el conflicto

\begin{tabular}{|c|c|}
\hline Tipología & Relato \\
\hline $\begin{array}{l}\text { Ataque contra medios } \\
\text { de transporte sanitario }\end{array}$ & $\begin{array}{l}\text { Alta conflictividad: } \\
\text { "Soy testigo de la intersección, quema y asesinato de la ambulancia del } \\
\text { centro de salud en el que yo trabajaba... la ambulancia la interceptan en } \\
\text { la vía San Carlos un comando guerrillero, asesinando a todos los } \\
\text { ocupantes, eso es retaliación porque en la zona en la que yo estaba que } \\
\text { era un municipio copado por los paramilitares y habia la asociación de } \\
\text { que todos los que estábamos allí éramos paramilitares o colaboradores" } \\
\text { (Profesional Académico). } \\
\text { Baja conflictividad: "Yo también iba en la ambulancia, y los } \\
\text { paramilitares, vestidos con chalecos, así normal nos pararon. ¿Para } \\
\text { dónde va la ambulancia?, ¿De dónde viene?, 'de una remisión', y ya } \\
\text { uno sigue y no más" (Vacunador). }\end{array}$ \\
\hline $\begin{array}{l}\text { Control o restricción del } \\
\text { tránsito de } \\
\text { medicamentos y/o } \\
\text { equipos }\end{array}$ & $\begin{array}{l}\text { Alta conflictividad: } \\
\text { "En ese tiempo una época que no podíamos ni pasar pastillas ni siquiera } \\
\text { para el dolor de cabeza. Por la violencia porque sí (por la desconfianza } \\
\text { entre ellos mismos) por decir en un retén le encontraban droga a uno } \\
\text { como una pastilla así fuera una pastilla entonces ya decían que uno les } \\
\text { pasaba droga a los otros a la guerrilla" (Víctima del Conflicto). } \\
\text { Baja conflictividad: No aplica. }\end{array}$ \\
\hline $\begin{array}{l}\text { Hurto de medicamentos } \\
\text { y/o equipos }\end{array}$ & $\begin{array}{l}\text { Alta conflictividad: } \\
\text { "Las brigadas eran de } 40 \text { o } 30 \text { días por río, entonces si nos agarraban de } \\
\text { los primeros dias, quedábamos muy de malas porque en ocasiones } \\
\text { querían que se les hiciera donación de medicamentos, de insumos, } \\
\text { entonces no se podía decir que no" (Enfermera Jefe). } \\
\text { Baja conflictividad: No aplica. }\end{array}$ \\
\hline $\begin{array}{l}\text { Restricción o } \\
\text { prohibición de la oferta } \\
\text { de servicios de salud }\end{array}$ & $\begin{array}{l}\text { Alta conflictividad: } \\
\text { "Hay zonas prohibidas y horarios prohibidos o tiempos prohibidos, por } \\
\text { ejemplo, en Baudó, de seis de la tarde a seis de la mañana no tenían } \\
\text { permiso [prestar servicios de salud], estaba por los diferentes grupos } \\
\text { armados, entonces limitaba la posibilidad de atender } \\
\text { urgencias" (Profesional Académico). } \\
\text { Baja conflictividad: No aplica. }\end{array}$ \\
\hline $\begin{array}{l}\text { Forzar la atención en } \\
\text { salud en condiciones } \\
\text { inadecuadas }\end{array}$ & $\begin{array}{l}\text { Alta conflictividad: } \\
\text { "Pues puede haber determinado tipo de presiones por parte de los } \\
\text { actores armados para que atiendan a su población... en algunas } \\
\text { circunstancias, uno también ha escuchado que algunos profesionales de } \\
\text { la salud han sido obligados a ir a estos espacios" (Médico). } \\
\text { Baja conflictividad: No aplica. }\end{array}$ \\
\hline
\end{tabular}

Fuente: elaboración propia. 
En el municipio de baja conflictividad solo se reportó la toma del centro de salud de la cabecera urbana por algunos miembros de un grupo armado, para exigir la atención a uno de sus cabecillas. Luego de este evento no se volvieron a presentar incidentes.

\section{Violaciones al secreto profesional}

Fueron pocos los participantes que narraron experiencias de violación al secreto profesional. Esta situación se presentó principalmente en el municipio de alta conflictividad, y estuvo relacionada con la práctica de los grupos armados de forzar al personal de salud a entregar información confidencial de algunos pacientes con patologías específicas o en casos de violencia sexual. Por otro lado, de manera ocasional se reportaron relaciones de parentesco entre miembros de los grupos armados y el personal de los servicios de salud. Por tal razón, algunas personas preferían no consultar, temiendo que su diagnóstico fuera revelado. En esos casos, las ONG y los programas de Naciones Unidas tenían mayor acogida y legitimidad. En el municipio de baja conflictividad no se reportó ningún caso de esta naturaleza asociado al conflicto armado, sino más bien en relación con la violencia de género y la vinculación de un médico del hospital a esta agresión.

\section{Recomendaciones sobre la atención y personal de salud en el post-acuerdo}

De acuerdo con sus experiencias y conocimiento, los participantes realizaron recomendaciones sobre cómo se podría mejorar el acceso a la salud, la prestación de los servicios y las condiciones del personal de salud, en el post-acuerdo. En el caso del municipio de alta conflictividad, se hace un llamado a brindar servicios de salud acordes a los contextos, en los que se reconozcan las problemáticas de las ruralidades que han estado aisladas y que viven situaciones asociadas al conflicto armado. Generalmente, estas problemáticas comprenden la presencia de cultivos de uso ilícito, la ausencia de servicios públicos y las dificultades de movilidad para acceder a servicios en salud que solo se prestan en las zonas urbanas de los municipios. Esta demanda se vio reflejada en el acuerdo de paz firmado por las FARC y el Gobierno Nacional en 2016. En la fase de implementación del acuerdo se diseñó un Plan Nacional de Salud Rural que busca atender esas necesidades. Según los participantes, llevar a cabo este plan implica un gran reto en la construcción del tejido social comunitario y en la recuperación de la confianza con las instituciones del Estado. Este es un paso necesario para transformar los territorios y superar las brechas que ha dejado el conflicto. Ver la tabla 3. 
Isabel Cristina Garcés Palacio/ Sara Milena Ramos Jaraba/ Natalia Quiceno Toro/ Marlly Andrea García Jiménez/ et al.

Tabla 3 Recomendaciones sobre la atención y personal de salud en el post-acuerdo (Cont.)

\begin{tabular}{|c|c|}
\hline Tipología & Relato \\
\hline Trabajo intersectorial & $\begin{array}{l}\text { "Hay } 170 \text { municipios priorizados, es que el servicio de salud les } \\
\text { queda muy lejos; no es tan fácil y no necesariamente la respuesta } \\
\text { sea construir un puesto de salud, una respuesta puede ser si el } \\
\text { desarrollo vial fuera mucho mejor y si pavimentas la vía, se } \\
\text { reduce el tiempo del trayecto" (Funcionario del Gobierno). }\end{array}$ \\
\hline $\begin{array}{l}\text { Garantías políticas de } \\
\text { transición }\end{array}$ & $\begin{array}{l}\text { "Bueno, ordenar los problemas por categorías podría uno } \\
\text { organizar, puede uno estar desde el nivel político porque estamos } \\
\text { en un periodo de transición política e inestabilidad por el cambio } \\
\text { de gobierno, porque finalmente todos los actores han entendido } \\
\text { que el tema de salud es transversal independiente del nivel } \\
\text { político que se está manejando" (Funcionario de la ONU). }\end{array}$ \\
\hline $\begin{array}{l}\text { Implementar un modelo } \\
\text { de salud diferencial para } \\
\text { el área rural }\end{array}$ & $\begin{array}{l}\text { "Yo creo que los retos son los mismos si vamos a aplicar el } \\
\text { mismo sistema que funciona en las ciudades o que no funciona } \\
\text { en las ciudades o si vamos a generar una politica clara en cómo } \\
\text { ser eficientes en la atención en salud rural, eso lo debe responder } \\
\text { un Plan Nacional de Salud Rural que a mi juicio tiene que tener } \\
\text { una construcción muy parecida a lo que fue en algún momento el } \\
\text { Plan Decenal de Salud Pública" (Funcionario de la ONU). }\end{array}$ \\
\hline $\begin{array}{l}\text { Ejecutar acciones con } \\
\text { enfoque diferencial y } \\
\text { territorial }\end{array}$ & $\begin{array}{l}\text { "La política debe incluir las pluralidades, pensar en que no solo } \\
\text { hay un campesino, una embarazada, atravesar por género, etnia, } \\
\text { clase, discapacidad. Las acciones en salud no están pensadas } \\
\text { según el contexto" (Profesional Académica). }\end{array}$ \\
\hline $\begin{array}{l}\text { Mejorar la gestión de } \\
\text { los hospitales públicos }\end{array}$ & $\begin{array}{l}\text { "Trabajar en el acceso a los hospitales y en las ESE que existen y } \\
\text { en la gestión porque en los dos sitios están a punto de quebrar... } \\
\text { a lo mejor movilizar porque esas brigadas llegan a esos sitios } \\
\text { más remotos ayudaría, seguramente también en atención primaria } \\
\text { y en salud" (Funcionaria de una ONG). }\end{array}$ \\
\hline $\begin{array}{l}\text { Fortalecer la Atención } \\
\text { Primaria en Salud }\end{array}$ & $\begin{array}{l}\text { "La gente necesita servicios adecuados a sus condiciones } \\
\text { culturales, necesita acciones extramurales de atención primaria } \\
\text { en salud, por qué, porque la gente vive lejísimos" (Funcionaria } \\
\text { de la ONU). }\end{array}$ \\
\hline $\begin{array}{l}\text { Reconstrucción del tejido } \\
\text { social comunitario y } \\
\text { confianza con las } \\
\text { instituciones del Estado }\end{array}$ & $\begin{array}{l}\text { "Dentro del conflicto se rompe o se fragmenta el tejido social, } \\
\text { cómo puedo confiar en el que está al lado, cómo hago para } \\
\text { conocer el de allí. Necesitamos trabajar más fuertemente porque } \\
\text { en muchos casos ha habido desconfianza, desconfianza desde el } \\
\text { Estado, desconfianza en los gobernantes y desconfianza en las } \\
\text { acciones y yo creo que reparar esos vínculos es una de las cosas } \\
\text { más dificiles de abordar" (Profesional Académica). }\end{array}$ \\
\hline
\end{tabular}

Fuente: elaboración propia. 
Tabla 3 Recomendaciones sobre la atención y personal de salud en el post-acuerdo (Cont.)

\begin{tabular}{|c|c|}
\hline Tipología & Relato \\
\hline $\begin{array}{l}\text { Telemedicina y otros } \\
\text { sistemas de comunicación } \\
\text { con las comunidades }\end{array}$ & $\begin{array}{l}\text { "Pues mira, por ejemplo, estas zonas, independiente del conflicto } \\
\text { o no conflicto son zonas aisladas de dificil acceso, un tema clave } \\
\text { es el tema de comunicación, si estas comunidades tienen un } \\
\text { radio, un celular, alguna manera de comunicarse con el hospital } \\
\text { y, el hospital tiene alguna manera de enviar alguna lancha, un } \\
\text { helicóptero para trasladar embarazadas, niños graves, esto } \\
\text { mejoraria mucho el sistema... el contacto de un promotor de una } \\
\text { red de auxiliares, es un nivel general de comunicación" } \\
\text { (Profesional Académico). }\end{array}$ \\
\hline $\begin{array}{l}\text { Fortalecer la atención en } \\
\text { salud mental a población } \\
\text { afectada por el conflicto }\end{array}$ & $\begin{array}{l}\text { "El tema de salud mental, que no solamente afecta al grupo, la } \\
\text { mujer o al niño, sino que afecta a todo el grupo de convivencia } \\
\text { de la comunidad, es un tema muy sentido que se requiere } \\
\text { atención" (Funcionaria de ONU). }\end{array}$ \\
\hline $\begin{array}{l}\text { Generar mayores } \\
\text { incentivos para el trabajo } \\
\text { en zonas de conflicto y } \\
\text { postconflicto }\end{array}$ & $\begin{array}{l}\text { "La estrategia de talento humano, ustedes saben que la rotación } \\
\text { de personal en estas zonas es muy alta, generar los incentivos } \\
\text { adecuados para mantener el personal en estas zonas apartadas se } \\
\text { vuelve muy importante" (Profesional Académico). }\end{array}$ \\
\hline $\begin{array}{l}\text { Mejorar la formación de } \\
\text { los profesionales y } \\
\text { contextualizarla a las } \\
\text { realidades colombianas }\end{array}$ & $\begin{array}{l}\text { "Muchos de los problemas que tenemos en salud materna es } \\
\text { porque los estudiantes, los médicos no saben de salud sexual y } \\
\text { reproductiva y mientras están leyendo un Journal England de } \\
\text { salud materna, desconocen los contextos de la salud materna en } \\
\text { Colombia" (Funcionario de una ONG). }\end{array}$ \\
\hline $\begin{array}{l}\text { Formación técnica/ } \\
\text { tecnológica/ } \\
\text { profesional a pobladores } \\
\text { de zonas rurales }\end{array}$ & $\begin{array}{l}\text { "Se están formando técnicos en salud pública en estos } \\
\text { municipios, la idea es que sean personas de la región, se prioriza } \\
\text { eso también, precisamente para evitar la altisima rotación de } \\
\text { personas, además porque la apuesta del acuerdo de paz es } \\
\text { fortalecer las capacidades locales" (Funcionario de Gobierno) }\end{array}$ \\
\hline $\begin{array}{l}\text { Formar a los profesionales } \\
\text { de la salud en misión } \\
\text { médica y temas de } \\
\text { conflicto }\end{array}$ & $\begin{array}{l}\text { "De lo que nos hablaban era de los comportamientos que el } \\
\text { personal de salud debe tener cuando salen a una misión a prestar } \\
\text { servicios en las zonas rurales: que usted andando despacio en el } \\
\text { carro, con las ventanillas abajo, con las luces de adentro } \\
\text { prendidas si es de noche para que los actores los puedan } \\
\text { identificar y saber de quién se trata, que no se deben trasladar ni } \\
\text { paquetes, ni personas en los carros de misión médica, que se } \\
\text { debe andar con los emblemas bien visibles, que no se debe } \\
\text { atender gente armada ni uniformada... Tratábamos de ir siempre } \\
\text { los mismos para tratar de crear esa confianza de los diferentes } \\
\text { actores" (Gerente de Hospital/Enfermero). }\end{array}$ \\
\hline
\end{tabular}

Fuente: elaboración propia. 
En el municipio de baja conflictividad las sugerencias estuvieron ligadas a las problemáticas de salud que aquejan al país debido a la desfinanciación de los hospitales públicos.

Con relación al personal de salud, existen problemáticas estructurales que hacen parte del contexto nacional, como la contratación ilegal, la tercerización laboral y el no pago de salarios por la iliquidez de los hospitales (11). Estas circunstancias se presentan en los dos municipios estudiados y deben ser intervenidas. En este sentido, para los participantes la academia cumple un papel clave en la formación de los profesionales y técnicos de la salud. La academia tiene incidencia en la calidad de la atención a través de la humanización de los servicios, en la contextualización de la práctica médica según las realidades y problemas de salud locales (especialmente lo asociado a atención diferencial), y en la formación y capacitación de los estudiantes en Misión Médica.

Por otro lado, los participantes de manera sistemática exponen la importancia que tiene para las zonas más alejadas y con alta intensidad del conflicto armado, contar con personal altamente calificado, estable, y con mayores garantías laborales. En este sentido, existe una necesidad sentida de volver a la figura del "promotor de salud rural", capacitado para ejercer funciones de atención primaria en salud, además de cumplir un rol dentro del sistema general de seguridad social (Tabla 3). Esta figura puede ser una solución a la dificultad para la vinculación del personal de salud en los municipios con altos índices de conflicto armado, ya que es la propia población local la que recibe esta formación, pudiendo desempeñarse de manera idónea. En cualquier caso, se deben garantizar las condiciones de seguridad para ellos. 


\section{Discusión}

Dentro del estudio se reportaron barreras estructurales del sistema de salud y de las comunidades que, aunadas al conflicto, generaron mayores dificultades para el acceso y la prestación de los servicios de salud. Durante el conflicto se presentaron diferentes formas de restricción en la movilidad generadas por las dinámicas propias de la violencia en Colombia. Esto generó la suspensión de los servicios de salud extramurales en el municipio de alta conflictividad, además de dificultades de acceso a los servicios de salud y medicamentos. Se reportaron también transgresiones a la Misión Médica como infracciones contra la vida y la infraestructura, y violaciones al secreto profesional. En el post-acuerdo, los participantes recomendaron brindar servicios de salud acordes con los contextos locales, mejorar las condiciones laborales y la formación del personal de salud, incluyendo aspectos relacionados con el DIH y la Misión Médica.

Se identifica como problemática recurrente en los hospitales del municipio de alta conflictividad, la alta rotación del personal, afectando no solo la calidad, sino también el acceso de las poblaciones rurales a clínicas móviles o brigadas extramurales. Esto implica además la pérdida de continuidad en los procesos. La alta rotación se debe, en parte, a que el personal muchas veces no tiene las condiciones adecuadas para ejercer su profesión. Igualmente, algunos profesionales no son oriundos de las zonas y no están suficientemente contextualizados con las dinámicas territoriales, desconociendo incluso cómo debe ser su accionar en un lugar donde confluyen diferentes grupos armados. Adicionalmente, en zonas de alta conflictividad, el personal de salud arriesga su vida por atender a pobladores o diferentes actores armados (5). Estas situaciones originaron miedos generalizados en el gremio, lo que dificulta la contratación de personal cualificado para dicha asistencia e impacta la vida cotidiana y la salud de la población. Como consecuencia, la población alimenta desconfianza y poca credibilidad hacia la institucionalidad, haciendo más fácil el sometimiento a las normas impuestas por los actores armados. Esto, por supuesto, termina por profundizar la brecha entre la población rural y la urbana en cuanto al acceso a la salud. Se espera que este sea uno de los aspectos a ser mitigados con la aplicación del Plan Nacional de Salud Rural, el cual es el primer punto del acuerdo final para la terminación del conflicto (10). Se entiende que hay una intersección en los factores de vulnerabilidad de las poblaciones más aisladas y marginadas, y su acceso al pleno goce de sus derechos. Las inequidades imbricadas se vuelven factores de riesgo o estructurales que modifican las condiciones de salud de una población, permitiendo que, en muchos casos, se presenten enfermedades que en otras circunstancias serían evitables.

Por otro lado, los relatos de las continuas transgresiones a la Misión Médica en el municipio de alta conflictividad demuestran una clara violación a las normas internacionales que regulan las situaciones de conflictos armados, en especial las tipificadas en el II Convenio de Ginebra (2). 
En los últimos 15 años, han sido reportadas situaciones similares por el Comité Internacional de la Cruz Roja (CICR) y la Organización Mundial de la Salud en países como Ruanda, Somalia, Yemen, Siria y Afganistán. Aunque no se tiene información precisa sobre el aumento de estas infracciones, hay investigaciones que muestran la devastación y el impacto que estos conflictos han tenido en el personal médico y los sistemas de salud (11-13).

En Libia, un estudio mostró daños estructurales al 29\% de los centros de atención en salud, con casos de destrucción total o parcial. El mayor impacto que tuvieron durante el conflicto fue la falta de suministros médicos, la seguridad del personal y los problemas de comunicación (14). En la gobernación de Aden en Yemen se encontró que de seis hospitales, solo dos funcionaban, y de doce policlínicos, nueve eran funcionales. De las 616 farmacias, 351 no estaban operativas y 123 funcionaban parcialmente (12). Si bien nuestros hallazgos relacionados con el desabastecimiento de suministros, la falta de personal médico, cierre temporal de centros de salud en las áreas remotas, entre otras, fueron similares, no hubo testimonios de daños totales a la infraestructura. En términos generales, los hospitales públicos siguieron prestando atención en salud a la población más cercana al casco urbano. Esto demuestra que, tal como lo vimos en el municipio de baja conflictividad, la ausencia de conflicto armado favorece la creación y consolidación de una institucionalidad más sólida. En estos lugares, la prestación del servicio de salud puede ampliar su cobertura y permanencia en el tiempo.

En concordancia con los hallazgos de esta investigación, un estudio realizado en 2002 en el oriente del departamento de Antioquia encontró que las infracciones más frecuentes al personal médico han sido las amenazas contra funcionarios, seguidas de los actos de perfidia, los ataques a la vida y a la integridad (15). Más recientemente, al igual que nuestros resultados, otro estudio encontró graves casos de violencia contra el personal sanitario, incluyendo homicidio, lesiones, secuestro, acoso, amenazas, robos, intimidación, arresto y coerción para revelar información protegida. Otras infracciones se relacionan con amenazas por atender heridos de otros "bandos", además de forzar la atención de pacientes en condiciones inadecuadas y contra su voluntad (5).

Otro de los hallazgos de gran relevancia fue la falta de formación básica en DIH y Misión Médica en el personal involucrado en la atención en salud. Resultados similares fueron reportados en otra investigación en Colombia, en la cual solo 50\% de los estudiantes del sector salud próximos a graduarse tenía información sobre la protección que otorgan las normas del DIH, al tiempo que solo $36 \%$ había recibido información del conflicto como tema dentro de un curso académico (16). Lo anterior ha contribuido a la rotación del talento humano en salud en estas zonas, impactando negativamente la continuidad de la prestación de los servicios. Si bien en Colombia existen estrategias e incentivos salariales relacionados con el servicio social obligatorio en los municipios con altos niveles de conflicto armado, estos no han sido suficientes para lograr la cobertura deseada.

Los retos del post-acuerdo en materia de atención y personal en salud son apenas motivo de indagación en Colombia. Se requieren más estudios y esfuerzos decididos desde las directivas, para articular los esfuerzos que las organizaciones internacionales y nacionales llevan a cabo en 
el país, en materia de paz y salud. Esto, si bien es complejo en todos los procesos de transición, lo es más en Colombia cuando las políticas de paz se entienden como parte de las agendas gubernamentales.

Para terminar, se sugiere realizar estudios sistemáticos y nacionales, relacionados con las infracciones a la Misión Médica. Si bien, las experiencias expuestas en este artículo pueden mostrar un panorama de lo que sucede en un municipio de alta conflictividad, en relación con uno de baja, probablemente las afectaciones se replican en muchos de los territorios afectados por el conflicto armado. Igualmente, se considera que la dimensión de los daños o traumas individuales que han afectado las vidas de esos profesionales ha sido poco estudiada. Finalmente, se insiste en que este tipo de estudios comparativos, entre territorios de alta y baja conflictividad, permiten ver las diferencias en cuanto a las condiciones de infraestructura y servicios de salud, pero sobre todo en la estabilidad y bienestar del personal de salud.

\section{Conclusiones}

Son evidentes las diferencias encontradas en el municipio de alta conflictividad versus el de baja conflictividad, tanto en las condiciones en las que se encuentra la Misión Médica, como en el acceso y la prestación de servicios de salud. En el municipio de alta conflictividad se afectó la prestación del servicio, que no llegó a cesar totalmente gracias al compromiso de muchos funcionarios. No obstante, esto no es del todo deseable puesto que los programas no pueden depender de voluntades personales, sino de capacidades institucionales. La restricción a la movilidad impuesta por los grupos armados es uno de los aspectos que más afectación generó en la prestación del servicio, tanto para el desplazamiento de las personas, como para las actividades extramurales de la Misión Médica. En muchas ocasiones, esta última se vio en medio de la represión, amenaza, secuestro, retención de insumos, afectación de la infraestructura sanitaria, entre otras situaciones descritas por los profesionales de salud.

Si bien, algunos protocolos establecen lineamientos para la atención a la población víctima del conflicto armado, se requieren mayores y mejores herramientas para la atención en salud centradas en el personal que opera en dichas poblaciones. Además, es necesario que las políticas que se crean a nivel nacional puedan ser replicadas y transmitidas a los territorios, dada la necesidad de generar medidas de protección y capacitación al personal de salud.

En el municipio de alta conflictividad se encontraron barreras geográficas que afectaron directamente el acceso de la población a los servicios. Estas, si bien son condiciones de vulnerabilidad previa, se profundizan con la presencia de actores armados.

En el post-acuerdo, la presencia de varias ONG y organismos internacionales ha contribuido a mitigar los efectos del conflicto armado y a propiciar soluciones que, si bien son coyunturales, 
pueden ser aprendizajes relevantes para la aplicación de intervenciones en los municipios afectados.

\section{Agradecimientos}

Agradecimientos al personal de salud, funcionarios de $\mathrm{ONG}^{\prime}$ s, Naciones Unidas y en especial a las personas de la comunidad por contar sus experiencias durante el conflicto.

Financiación. Centro SickKids para la Salud Infantil Global en el Hospital for Sick Children, con costos de viaje y reuniones apoyados directamente por la Universidad Aga Khan y la Alianza para la Salud de la Madre, el Recién Nacido y el Niño (ASMRN). Como coordinador del Consorcio BRANCH (Bridging Research \& Action in Conflict Settings for the Health of Women \& Children), el Centro SickKids para la Salud Infantil Global ha recibido financiación para actividades de investigación del BRANCH del Centro Internacional para el Desarrollo de Investigaciones (IDRC), la Agencia Noruega de Cooperación para el Desarrollo (NORAD), la fundación Bill \& Melinda Gates, UNICEF. La Universidad Aga Khan ha recibido fondos para las actividades del BRANCH de la Fundación Family Larsson-Rosenquist. La Universidad de Antioquia proveyó financiación para los investigadores en Colombia.

\section{Referencias}

1. CMH. ¡BASTA YA! Colombia: Memorias de guerra y dignidad. Bogotá: Imprenta Nacional, 2013.

2. Geneva Convention. Geneva Convention for the amelioration of the condition of the wounded and sick in armed forces in the field. Geneva 1949. http://www.tc.gob.pe/tratados/uni_DDHH/instru_alca_es pecifi_uni/Derecho_humanitario/conve_ginebra2.pdf.

3. Colombia. Ministerio de Salud. Resolución 1020 de 2002 (agosto 5) Por la cual se dictan medidas para la protección de la Misión Médica. Bogotá: Ministerio de Salud y Protección Social; 2002.

4. Colombia. Ministerio de Salud y Protección Social. Resolución 4481 de 2012 ( 28 diciembre) Por la cual se adopta el Manual de Misión Médica y se establecen normas relacionadas con la señalización y divulgación de su Emblema. Bogotá: Ministerio de Salud y Protección Social; 2012.

5. Urrego, DZ. Conflicto armado en Colombia y misión médica: narrativas médicas como memorias de supervivencia. Rev. Fac. Med. 2015; 63(3): 377-88. https://doi.org/10.15446/revfacmed.v63n3.452 09

6. Boletín temático. Observatorio del Programa Presidencial de Derechos Humanos y DIH No. 3, enero de 2005. http://historico.derechoshumanos.gov.co/Observatorio/Publicaciones/Paginas/2005.aspx.

7. Comité Internacional de la Cruz Roja. Situación Humanitaria informe de actividades Colombia 2012 [Internet]. 2013. Disponible en: https://www.icrc.org/es/doc/assets/files/2013/colombia-report-2012 .pdf.

8. Galeano Marin ME. Estrategias de investigación social cualitativa: el giro de la mirada. Medellín, Colombia: La Carreta; 2004. 
Isabel Cristina Garcés Palacio/ Sara Milena Ramos Jaraba/ Natalia Quiceno Toro/ Marlly Andrea García Jiménez/ et al.

9. Krippendorff, K. Content Analysis: An Introduction to Its Methodology. 2ndEdition. Thousand Oaks, CA: Sage Publications; 2004. 413 p.

10. Acuerdo Final. (2016). Acuerdo Final para la Terminación del Conflicto y la Construcción de una Paz Estable y Duradera. Bogotá, Presidencia de la República.

11. World Health Organization (WHO). Servicios médicos y de salud en tiempos de conflicto armado. Ginebra: Consejo Ejecutivo; 1994. Report No. EB95/24.

12. Sallami Z, Kassim Y, Selvaraj J, Parry E, Winter G. Impact of the Armed Conflict of 2015-2016 in Aden on health services and the availability of medicines. Health. 2017;09(04): 685-696. https://doi.org/ 10.4236/health.2017.94049

13. Betsi NA, Koudou BG, Cissé G, Tschannen AB, Pignol AM, Ouattara Y, et al. Effect of an armed conflict on human resources and health systems in Côte d'Ivoire: Prevention of and care for people with HIV/ AIDS. AIDS Care. mayo de 2006;18(4): 356-365. https://doi.org/10.1080/09540120500200856

14. Mohamed AD, Abdallah E-B, Aghnaya AD. The assessment of efficiency and coordination within the Libyan health care system during the armed conflict-2011. Clinical Epidemiology and Global Health. 2016;4: 120-127. https://doi.org/10.1016/j.cegh.2015.07.004

15. Escobar ML, Bustos GM, Restrepo JE et al. Conocimiento y aplicación del Derecho Internacional Humanitario y la Misión Médica en los servicios de urgencias del oriente antioqueño. Iatreia. 2003;16(4): 263-272. http://www.scielo.org.co/scielo.php?script=sci_arttext\&pid=S0121-07932003 $000400001 \& \operatorname{lng}=$ en.

16. Laverde LA, López JI, Ochoa AL. Sector Salud, conflicto armado colombiano y Derecho Internacional Humanitario. Invest Educ Enferm. 2004;22(2): 62-75. 2966-Article Text-9657-1-10-20091120.pdf

Notas

* $\quad$ Artículo de investigación. 